\title{
Management of Kennedy Class I Partial Edentulism by Removable Cast Partial Denture: A Case Report
}

\author{
NB Jamayet ${ }^{1}$, U Habiba ${ }^{2}$, SZE Zai ${ }^{3}$, S Hossain ${ }^{4}$
}

\begin{abstract}
Background: Distal extension edentulism can affect the patient's ability to function as a dentate person. A well-made removable partial denture that has appropriate extensions, borders and ridge-to-dentition relationship will benefit the partially edentulous patient by providing increased comfort and improved dental function. This article described a patient who had a bilateral distal extension removable partial denture in both upper and lower arch. The prosthesis showed a successful rehabilitation with proper function and aesthetics.
\end{abstract}

Methods: A Kennedy class I bilateral free end saddle edentulous arch in both upper and lower arch was rehabilitated with removable cast partial denture. The case had the reasonable amount of crown: root ratio for the remaining abutment teeth for placement of metal framework, occlusal rest and cast clasp.

Results: The delivered denture showed proper sitting of occlusal rest and cast clasp. Retention and stability was acceptable. Occlusion had achieved its desired occlusion scheme.

Conclusion: Removable cast partial denture is a suitable option for the rehabilitation of partially edentulous case if the case has such amount of abutment teeth remaining with healthy periodontal condition.

Keywords: Removable cast partial denture, rest, cast clasp, abutment.

\section{Introduction}

Restoration of the lost or missing tooth/teeth is one of the challenging tasks for a clinician among various possible treatment modalities. The optimum restoration should fulfill the basic requirements of any prosthesis.

1. Dr. Nafij Bin Jamayet, BDS, Grad Dip Clin Sc in OMFP, MPH, M Sc in OMFP Assistant Professor \& Head of The Department Department of Dentistry International Medical College \& Hospital (Proposed Dental Unit) Gazipur

2. Dr. Umma Habiba, BDS. PGT, Registrar, Department of Dentistry, International Medical College \& Hospital, (Proposed Dental Unit) Gazipur

3. Dr. Sumaiya Zabin Eusuf Zai, BDS, MPH, Lecturer, Department of Public Health, Northern University, Dhaka

4. Dr. Sharafat Hossain, BDS, M Sc in Prosthodontics, Assistant Professor, Department of Prosthodontics, University Dental College \& Hospital, Dhaka

\section{Address of Correspondence:}

Dr. Nafij Bin Jamayet, Assistant Professor \& Head of The Department, Department of Dentistry, International Medical College \& Hospital (Proposed Dental Unit), Sataish, Tongi, Gazipur.

E-mail: dr.nafij@gmail.com
Restoration of partially edentulous arches is not an easy task for a clinician where the clinician has to give due consideration in fulfilling the necessary requirement of the patients. Restoration of partially edentulous arches by fixed partial dentures is the treatment of choice by any patient but may not be the desirable option in most of the situations. In most of the circumstances, partial dentures may not contribute greatly to mastication; masticatory efficiency of the fixed partial dentures will be considerably superior compared to the removable partial dentures ${ }^{1}$. Most of the partially edentulous arches exhibit tipped, rotated and extruded teeth, less space between the edentulous area and the remaining natural teeth, attrited teeth, teeth with short clinical crowns which require an extensive knowledgeable planning and fore-thought to a successful outcome as it is in practice of removable partial prosthodontics ${ }^{2}$. This case report describes a simple but as yet an effective method of restoration of partially edentulous arches with removable cast partial denture.

\section{Case Report}

A Kennedy class 1 bilateral free end saddle in both upper and lower arch was presented in a out patient Department at Maxillofacial prosthetic service, Mahidol University, Bangkok, Thailand in October, 2011.

The intra oral picture and mounted diagnosed casts showed sufficient space between the edentulous space and the opposing remaining natural teeth (Fig. 1). 

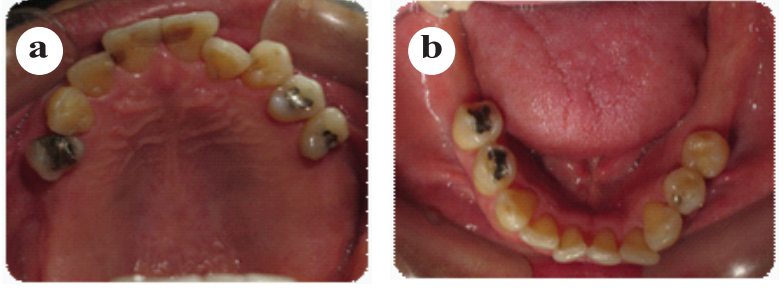

Fig 1: Intra oral view, a. upper, b. lower
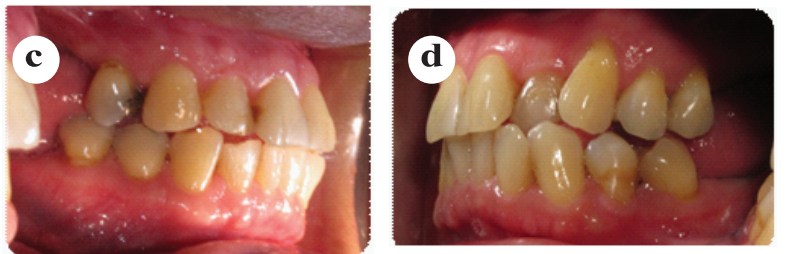

Fig 1: c. right lateral view \& d. left lateral view

The patient was planned to rehabilitate with maxillary and mandibular cast removable partial denture (RPD) .Diagnostic models were analyzed and were surveyed. Mock up mouth preparations were done on the diagnostic models and the desired preparations were executed on the teeth intraorally in the patient's mouth. A check model was fabricated to analyze the mock up preparation. Final mouth preparations were made in the patient's mouth and final impressions were made using polyether elastomeric impression material. Master cast was fabricated using high strength stone. Master cast surveying procedure was carried out to block the under cuts and refractory casts were made using phosphate bonded investment material (Fig. 2).
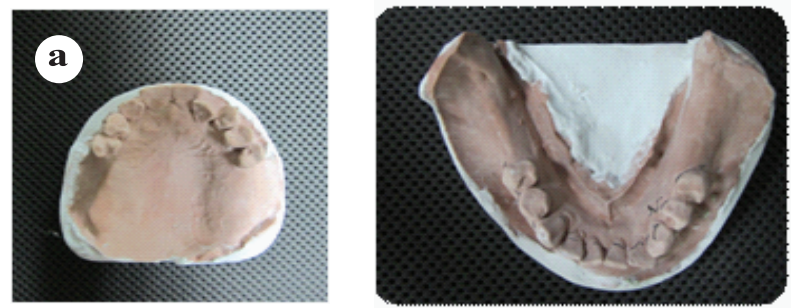

a. Master cast upper \& lower
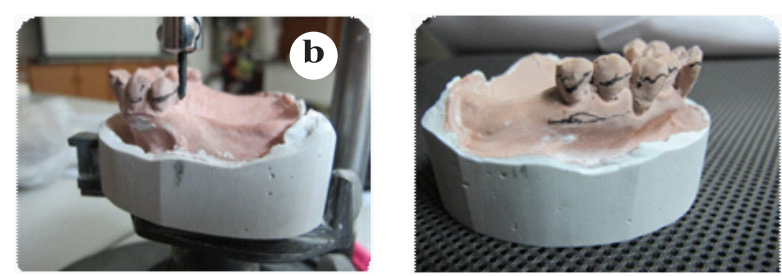

b. Survey tool with cast \& Surveyed cast

Fig 2: (a) Master cast \& (b) Survey of the cast.
Wax pattern of cast partial denture was made using inlay casting wax. For the design of RPD (Fig. 3), rests were placed on tooth no 13, 14, 23, 25 for upper \& in lower on 35 and 45 . For major connector we used palatal coverage for upper \& lingual plate for lower. Cast clasps were used on abutment tooth no. 14,25 for upper and 35,45 for lower, the reason that we used cast clasp on this long distal extension base because abutment teeth had good bony support, periodontal health status, with good crown root ratio.

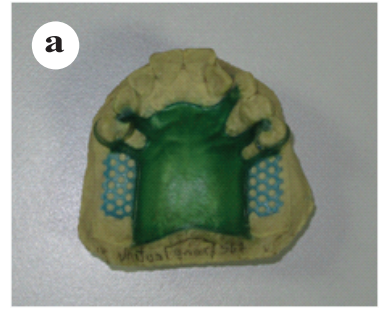

a. upper framework design

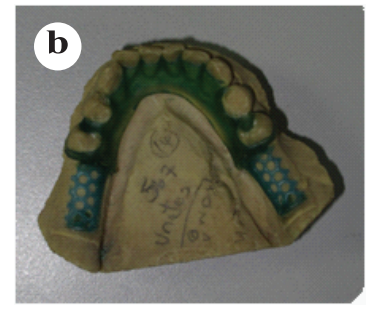

b. lower framework design
Fig: 3 Removable partial denture metal framework design. (a) Upper Arch (b) Lower Arch

The refractory model with the wax pattern was invested and casting procedure was carried out. The casted cast partial denture was finished and polished in the conventional manner. The metal framework was tried in the patient's mouth for proper seating of rests and clasps (Fig. 4a,b,c,d).

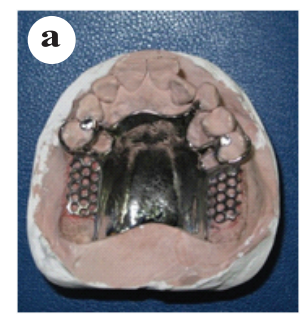

a.Metal framework in upper cast

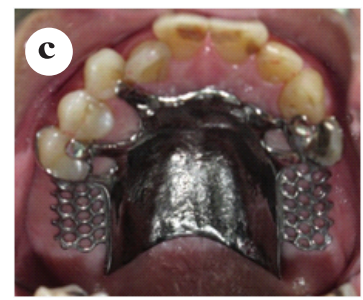

c.Metal framework try in upper jaw

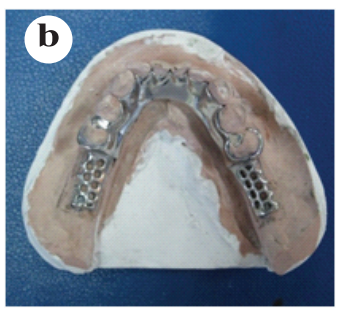

b.Metal framework in upper cast

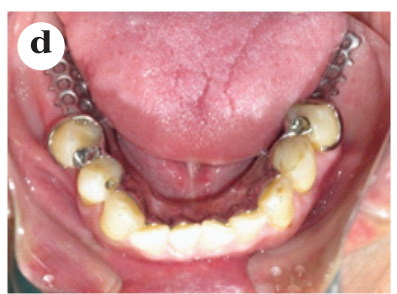

d.Metal framework tryin lower jaw
Fig 4 : Metal framework try in a. upper cast, b. lower cast. c. upper jaw and d. lower jaw. 
Bite block try in was carried out and bite registration was taken. For this case we have chosen bilateral balancing occlusal scheme. Artificial teeth were arranged in semi adjustable articulator (Fig.5a,b,c).

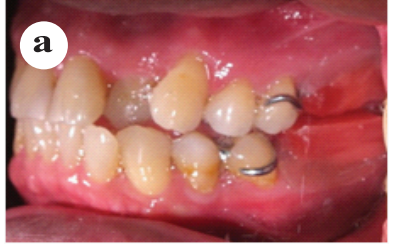

a.Bite block try in mouth

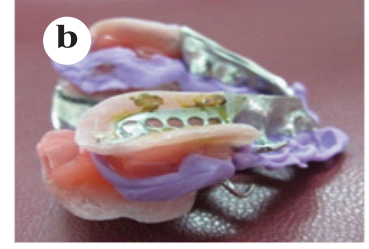

b..Bite registration

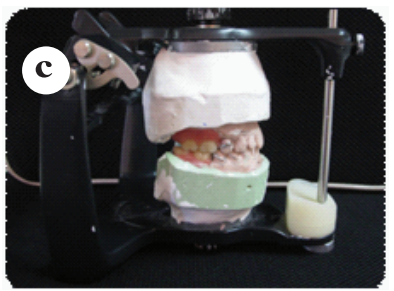

c.Artificial teeth setting in semi-adjustable articulator

Fig 5: a. bite block try in, b. registration \& c. artificial teeth setting.
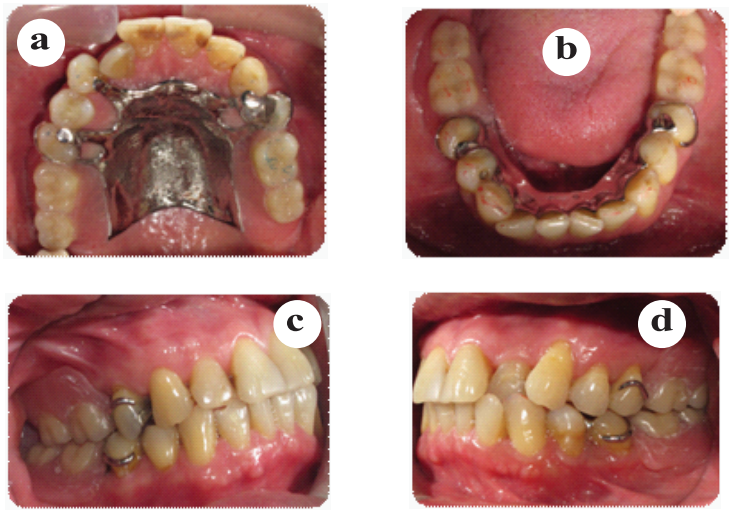

Fig 6: Intra oral view of Inserted denture upper, lower, right \& left lateral view.

The finished flangeless cast partial denture was tried in the patient's mouth and adjustments were made for lateral excursions (Fig.6a,b,c,d).

\section{Discussion}

General considerations for cast partial denture are reviewed on basis of soft tissue variables $\&$ hard tissue variables and special consideration for distal extension. $^{3}$

\section{Considerations for Soft Tissue Variables}

Soft tissue anatomy such as frenal attachments and vestibular depth can affect the choice of major connectors and direct retainers.
Characteristics of the soft tissues, such as undercuts and tissue compressibility of attached mucosa, may also affect design decisions. These aspects of the tissue need to be identified intra orally, since they cannot be frequently determined solely on the basis of a diagnostic cast.

\section{Considerations for Hard Tissue Variables}

The opposing occlusion, significant abutment mobility's, the access to embrasures, presence of rotations, the positions of tooth undercuts and the presence of restorations can all influence the selection of direct retainers. The presence of tori can affect major connector selection.

\section{Special consideration for Distal Extension Base}

1. Use stress releasing direct retainers in distal extension cases. There are three axes of rotation for these partial dentures. If abutment teeth are locked into the frameworks they can be torqued in many directions. Stress-relieving clasps allow for some release of the teeth to minimize torquing potential.

2. Rotational movements increase with length of span of the distal extension. Also as arm flexibility increases, resistance to lateral displaceability decreases. Therefore, it is important to use maximum coverage of the edentulous ridge to reduce the degree of lateral movement. This is particularly effective when there are large, broad ridges which tend to provide greater resistance to horizontal movements.

3. So for the stress releasing to the direct retainers and prevent the rotational movements of denture, alter cast impression technique should be applied to resolve the problem. ${ }^{4}$

\section{Conclusion}

Removable cast partial dentures are suitable options for the rehabilitation of partially edentulous cases if the cases have such amount of abutment teeth remaining with periodontal condition. More ever sometimes it is better than long span fixed bridge restorations. With proper fitting of cast partial denture patient can find his or her lost occlusion and continue their life with proper nutrition.

\section{References}

1. Osborne J, Why make partial dentures; Partial Dentures: 4th edn, CBS Publishers 1985.

2. Grasso JE, Miller EL, Removable partial Prosthodontics: 3rd edn, Mosby Publication.

3. Ahmad I. General considerations in cast partial dentures. Practical procedures and aesthetic dentistry, 2002; 14(3) 233-238.

4. Kurtzman GM, Schneider AL. A technique for Alter cast impression technique method. Dent Today, 2006; 25(1): 94-95. 